Research Article

\title{
Unraveling the Molecular Mechanisms of Fructus Anisi Stellati as a Remedy for Infantile Colic by Network Pharmacology
}

\author{
Xingyu $\mathrm{Li} \mathbb{D}^{1,2}$ and Yan $\mathrm{Xu} \mathbb{D}^{1}$ \\ ${ }^{1}$ Department of Chemistry, Cleveland State University, Cleveland, OH 44115, USA \\ ${ }^{2}$ College of Science, Yunnan Agricultural University, Kunming 650201, China \\ Correspondence should be addressed to Xingyu Li; lixingu1225@hotmail.com and Yan Xu; y.xu@csuohio.edu
}

Received 15 June 2020; Revised 4 December 2020; Accepted 10 December 2020; Published 18 December 2020

Academic Editor: Zihu Guo

Copyright (c) 2020 Xingyu Li and Yan Xu. This is an open access article distributed under the Creative Commons Attribution License, which permits unrestricted use, distribution, and reproduction in any medium, provided the original work is properly cited.

\begin{abstract}
Fructus anisi stellati (FAS) is an anise-scented star-shaped fruit from Illicium verum tree. It is commonly consumed in many cultures as food and medicine, particularly as a remedy for infantile colic (IC). The elucidation of molecular mechanisms of action would contribute to the understanding of the traditional therapy of FAS and help to guide the preclinical and clinical study of this herb. The aim is to investigate the key therapeutic compounds of FAS and to explore the underlying molecular mechanisms of FAS therapy. The chemical compounds of FAS were obtained through data mining on TCMSP and ADME screening, and the common targets of the FAS compounds and the IC-correlated diseases were obtained from PharmMapper, GeneCards, and OMIM databases. GO and KEGG databases were used for molecular function and pathway enrichment. Cytoscape was used for network construction and analysis. SystemsDock was used for molecular docking. Three key compounds (i.e., quercetin, luteolin, and kaempferol), 19 targets, 7 molecular pathways, and 12 IC-correlated diseases were identified to be involved in the molecular mechanisms of FAS for the treatment of IC. This work showed that three therapeutic modules were primarily engaged in the molecular mechanisms of FAS for IC therapy, including the inhibition of inflammatory reactions, stimulating immunoglobulin A $(\operatorname{IgA})$ production in the gastrointestinal tract, and enhancing the secretion of digestive enzymes.
\end{abstract}

\section{Introduction}

Infantile colic (IC) describes a symptom complex of excessive and inconsolable crying in babies that is a common phenomenon in infancy. Although IC is usually a selflimiting condition, it is a source of extreme distress for the infant, parents, family, and health-care professionals [1]. At present, the specific focus or nature of IC remains unclear. Various etiologic factors have been proposed to contribute to this disorder, including environmental, psychosocial, physical, neurodevelopmental, nutritional, and gastrointestinal factors. Although no firm conclusions can be drawn paving the way for a multifactorial explanation for this entity, as the academic purposes the Rome IV criteria define IC as a functional gastrointestinal disorder from birth to five months of age $[1,2]$. Several possible gastrointestinal factors have been suggested to contribute to the pathophysiology of
IC including cow's milk protein intolerance, gastroesophageal reflux, excessive intestinal gas, lactose intolerance, and gut hormones [1]. Herbal medicine is a popular strategy applied for the management of IC caused by gastrointestinal factors $[3,4]$, which contain multiple components such as fennel [5].

Fructus anisi stellati (FAS) is an anise-scented starshaped fruit of Illicium verum Hook. f. belonging to the Schisandraceae family [6], according to the plant list (http://www.theplantlist.org), commonly known as star anise or Chinese star anise, and is an aromatic mediumsized evergreen tree, native to northeast Vietnam and southwest China [7], and distributed in North America, Atlantic region, and tropical and subtropical zones of Asia [8]. FAS is commonly known as being safe and nontoxic when consumed as both food and medicine in many cultures [9]. It has traditionally been used as a flavor 
ingredient in daily cuisine recognized in China as far back as 100 B.C. It has also been used in medicine throughout Asia and North America for many illnesses [10], in which it has been widely used to treat childhood colic, abdominal pain, colitis, diarrhea, and bloating [11]. While FAS is commonly used, and its impact is appealing as an important traditional Chinese medicine (TCM), the potential molecular mechanisms of its effect on IC were not evident. Multicomponent and multitarget are the significant features of TCM, which have made the molecular mechanism analysis complex and challenging. Fortunately, network pharmacology has been proven to be a suitable method to explore the underlying mechanism between TCM and known targets systematically. Based on the interaction among active components of TCM and their protein targets, as well as relevant biological functions and pathways, a network pharmacology study enables us to investigate the possible molecular mechanisms of a TCM to a particular disease [12].

In the current studies, the targets of FAS active compounds and IC-correlated pathways were carefully evaluated based on the network pharmacology to systematically explore the prospective targets and molecular mechanism and provide a hypothesis for IC therapeutic research and clinical study.

\section{Materials and Methods}

2.1. Screening Candidate Compounds in FAS. Forty-nine compounds of FAS (Table S1) were obtained from the Traditional Chinese Medicine Systems Pharmacology (TCMSP) database (http://lsp.nwu.edu.cn/tcmsp.php) which is a distinctive Chinese herbal medicines pharmacology platform that captures drug, target, and disease interactions [13]. Then, the candidate compounds were screened using two ADME (short for Absorption, Distribution, Metabolism, and Excretion) models, including oral bioavailability $(\mathrm{OB})$ and drug-likeness (DL). The threshold values for these screening models are set to $\mathrm{OB} \geq 30 \%$ and $\mathrm{DL} \geq 0.18$, respectively [14].

2.2. Prediction Targets of Candidate Compounds. The targets of FAS compounds were retrieved from the TCMSP and searched in the UniProt (http://www.uniprot.org) database for human-correlated protein codes. Together, an online target prediction platform PharmMapper (http:// lilab-ecust.cn/pharmmapper/) was also used to retrieve the targets of FAS compounds with a "fit score" > 4 [15].

2.3. Target Genes of IC-Correlated Diseases. The target genes of IC-correlated diseases were gathered from the GeneCards database (https://www.genecards.org/) [16] and the OMIM database (http://www.omim.org/) [17]. The search keywords were "colitis OR diarrhea OR "lactose intolerance" OR "infantile colic" OR "abdominal pain" OR "inflammatory bowel disease (IBD)" with the relevance score $>5$.
2.4. Common Targets of the FAS Compounds and IC-Correlated Diseases. The common targets of the FAS compounds and IC-correlated disease were identified by Venn analysis (http://bioinfogp.cnb.csic.es/tools/venny/). These common targets were further cross-referenced with David (https:// david-d.ncifcrf.gov/) and PDB (http://www.rcsb.org/pdb/ gene/) [18]. Cytoscape software (https://cytoscape.org/ ,version.3.8.0) was used to construct and visualize the interaction network of FAS compounds and the targets of IC.

2.5. Protein-Protein Interaction Data. The common targets were used to construct the protein-protein interaction (PPI) network using the String database (https://string-db.org/) with setting up multiple proteins and Homo sapiens, and confidence scores $>0.7$.

2.6. GO and KEGG Enrichment Analyses. To systematically elucidate the molecular interaction network of common targets, Gene Ontology (GO) molecular function and Kyoto Encyclopedia of Genes and Genomes (KEGG) pathway enrichment analyses were performed using $R$ (version 3.5.2) software packages with $p<0.05$ (http://www.bioconductor. org/) [19]. The significant molecular functions and pathways of the target genes were extracted.

2.7. Networks Construction and Analyses. To visualize and analyze various relationships among FAS, active compounds, targets, pathways, and diseases, Cytoscape was used to construct and analyze networks, including compoundstargets (C-T) interaction network and compounds-targetspathways-diseases (C-T-P-D) interaction network. The Cytoscape plugin Network Analyzer was used for network topological analysis.

2.8. Molecular Docking Simulation. Molecular docking was performed to confirm the binding properties of active compounds and key IC-correlated targets using SystemsDock [20].

\section{Results and Discussion}

3.1. The Workflow. A schematic diagram of the present study is shown in Figure 1. Firstly, the common targets were identified between the predicted targets of the active compounds and the IC-correlated genes. Then, the common targets were subjected to enrichment analyses for protein molecular functions and molecular pathways. Furthermore, the network between compounds, targets, pathways, and diseases was constructed to analyze the underlying molecular mechanism. Finally, molecular docking was performed to evaluate the binding properties between active compounds and targets, and supporting evidence to the proposed molecular mechanisms was provided.

3.2. Candidate Compounds in FAS. There were eight candidate compounds, including five (mairin, luteolin kaempferol, (+)-catechin, and quercetin) which passed the 


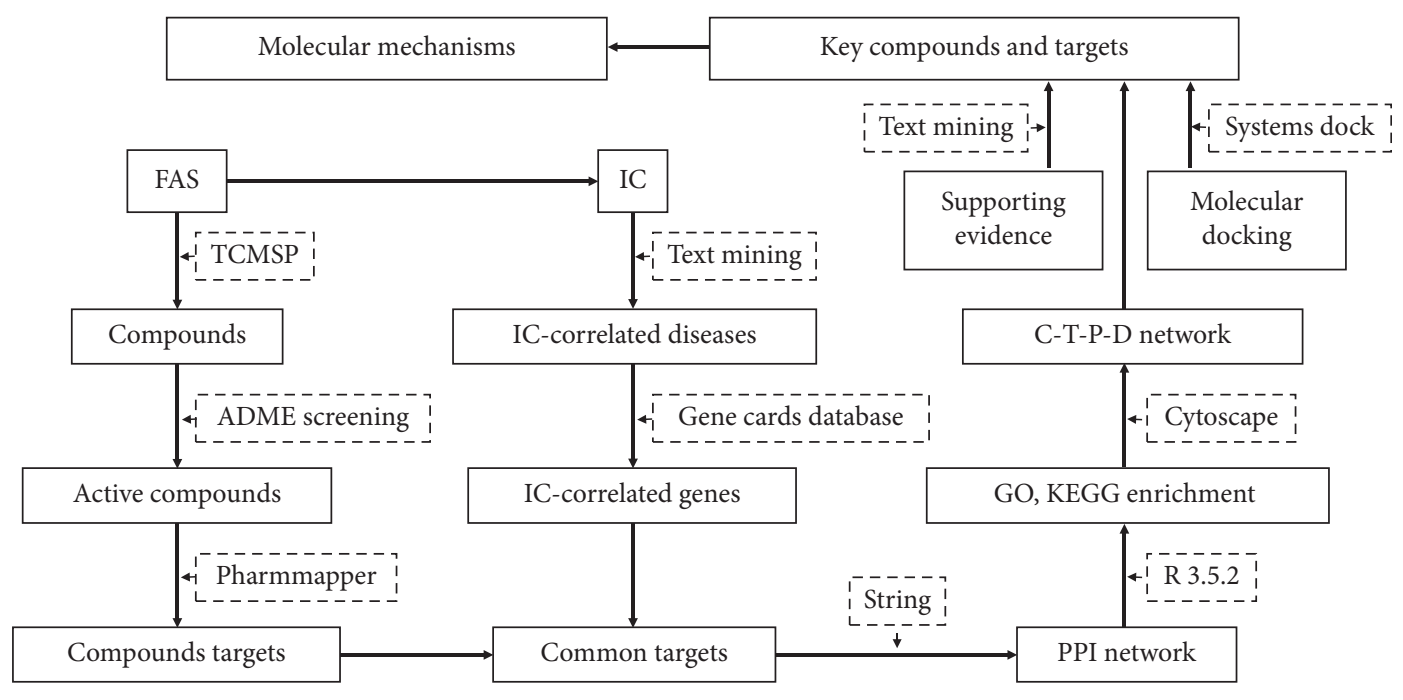

Figure 1: The workflow of the network pharmacology study of FAS.

ADME screening criteria and three (anethole, salicylic acid, and shikimic acid) selected based on the reported biological activities. For example, anethole, a type of aromatic compound that generally exists in nature as one of the essential oils, had a significant relaxing effect on tracheal and ileal smooth muscles, anti-inflammatory property, and rapid absorbability when orally administered [21]. Their chemical structures and ADME parameters were shown in Figure 2 and Table 1, respectively.

3.3. Common Targets of Candidate Compounds and IC. Based on the 8 candidate compounds, a total of 250 putative targets were retrieved from PharmMapper and TCMSP after removing duplicates (Table S2). There were 1,074 IC-correlated genes obtained from the GeneCard database and OMIM after removing duplicates (Table S3). Between the putative targets of the candidate compounds and IC-correlated genes, 92 common targets were identified (Table S4).

3.4. PPI Network of Common Targets. The common targets were used to construct the PPI network using String. Among the 92 common targets, 86 (Table S5) were associated with each other with a minimum of two connections and a confidence score $>0.7$, which were considered as significant targets of IC. As shown in Figure 3, the PPI network had 86 nodes and 779 edges. The larger the nodes are or the more the edges are, the higher the degree of centrality the nodes have and the more important the nodes are in the network. The biochemical classifications of these 86 significant targets include enzymes, immune system, cytokine, and transcription factors (Figure 4(a)). Among them, 38.4\% of 86 targets are enzymes including 14 hydrolases, 8 oxidoreductases, 6 transferases, 2 kinases, 1 isomerase, 1 ligase, and 1 lyase (Figure 4(b)). These enzymes play critical functions in biological processes. For example, gastric and pancreatic lipases play the main role in gastrointestinal digestion of nutritional fat [11], and about $40 \%$ of infants with IC suffered from a lactase deficiency [22].
3.5. Compound-Target (C-T) Network Analysis. The eight candidate compounds and 86 significant targets were used to construct a C-T network using Cytoscape (Figure 4(c)). The network consists of 96 nodes (86 targets, 8 compounds, 1 plant, and 1 disease) and 260 edges, of which 166 edges are formed between the compound and the target. According to the ranking of centrality degree, the top 3 ranked compounds were quercetin, luteolin, and kaempferol with degree of centrality values of 69,39 , and 29 , respectively. These 3 compounds bound to 76 important targets accounted for $88.4 \%$ of the total targets. These 3 compounds belong to a class of plant secondary metabolites known as flavonoids with various pharmacological activities [23]. Therefore, quercetin, luteolin, and kaempferol were considered as the key active compounds, and the 76 targets were considered as targets for further KEGG and GO enrichment analyses.

3.6. Enrichment Analyses and Therapeutic Modules. GO enrichment analysis was performed on 76 targets and 102 GO molecular functions were obtained $(p<0.05)$ (Table S6). The $102 \mathrm{GO}$ molecular functions were found to belong to 6 functional categories including 53 binding activities, 26 catalytic activities, 12 molecular function regulators, 8 transcription regulator activities, 2 antioxidant activities, and 1 molecular transducer activity.

KEGG enrichment analysis was also performed on 76 targets and resulted in 113 molecular pathways $(p<0.05)$ (Table S7). By searching the KEGG database, 288 diseases were found (Table S8). Among these findings, 7 molecular pathways (Table 2) and 12 diseases were correlated with IC (Table 3), which led to three therapeutic modules for the treatment of IC-correlated diseases.

Module I consisted of four pathways (i.e., hsa04973, hsa00052, hsa00500, and hsa04972) related to carbohydrate and fat digestion, absorption, and metabolism. For examples, hsa04973 is associated with congenital glucose-galactose malabsorption (H01261); and hsa00052 is related to galactosemia (H00070), congenital lactase deficiency 
<smiles>O=c1c(O)c(-c2ccc(O)cc2)oc2cc(O)cc(O)c12</smiles>

Kaempferol<smiles>O=c1cc(-c2ccc(O)c(O)c2)oc2cc(O)cc(O)c12</smiles>

Luteolin<smiles>O=c1c(O)c(-c2ccc(O)c(O)c2)oc2cc(O)cc(O)c12</smiles>

Quercetin<smiles>O=C(O)C1=C[C@@H](O)[C@H](O)[C@H](O)C1</smiles>

Shikimic acid<smiles>O=C(O)c1ccccc1O</smiles>

Salicylic acid<smiles>Oc1cc(O)c2c(c1)O[C@H](c1ccc(O)c(O)c1)[C@H](O)C2</smiles>

(+)-Catechin<smiles>C=C(C)[C@H]1CCC2(C(=O)O)CC[C@]3(C)[C@@H](CC[C@H]4[C@@]5(C)CC[C@H](O)C(C)(C)[C@@H]5CC[C@]43C)[C@@H]12</smiles>

Mairin<smiles>C/C=C/c1ccc(OC)cc1</smiles>

Anethole

FIGURE 2: The chemical structures of eight selected bioactive compounds of FAS.

TABLE 1: Selected bioactive compounds of FAS and their ADME parameters.

\begin{tabular}{|c|c|c|c|c|}
\hline No. & Compounds & OB $(\%)^{\mathrm{a}}$ & $\mathrm{DL}^{\mathrm{b}}$ & $\mathrm{HL}(\mathrm{h})^{\mathrm{c}}$ \\
\hline 1 & Mairin & 55.38 & 0.78 & 8.87 \\
\hline 2 & Luteolin & 36.16 & 0.25 & 15.94 \\
\hline 3 & Kaempferol & 41.88 & 0.24 & 14.74 \\
\hline 4 & $(+)$-Catechin & 54.83 & 0.24 & 0.61 \\
\hline 5 & Quercetin & 46.43 & 0.28 & 14.40 \\
\hline 6 & Anethole & 32.49 & 0.03 & 1.68 \\
\hline 7 & Salicylic acid & 32.13 & 0.03 & 12.00 \\
\hline 8 & $(-)$-Shikimic acid & 46.24 & 0.04 & 11.18 \\
\hline
\end{tabular}

${ }^{\mathrm{a}} \mathrm{OB}$, oral bioavailability, ${ }^{\mathrm{b}} \mathrm{DL}$, drug-likeness, and ${ }^{\mathrm{c}} \mathrm{HL}$, half-life.

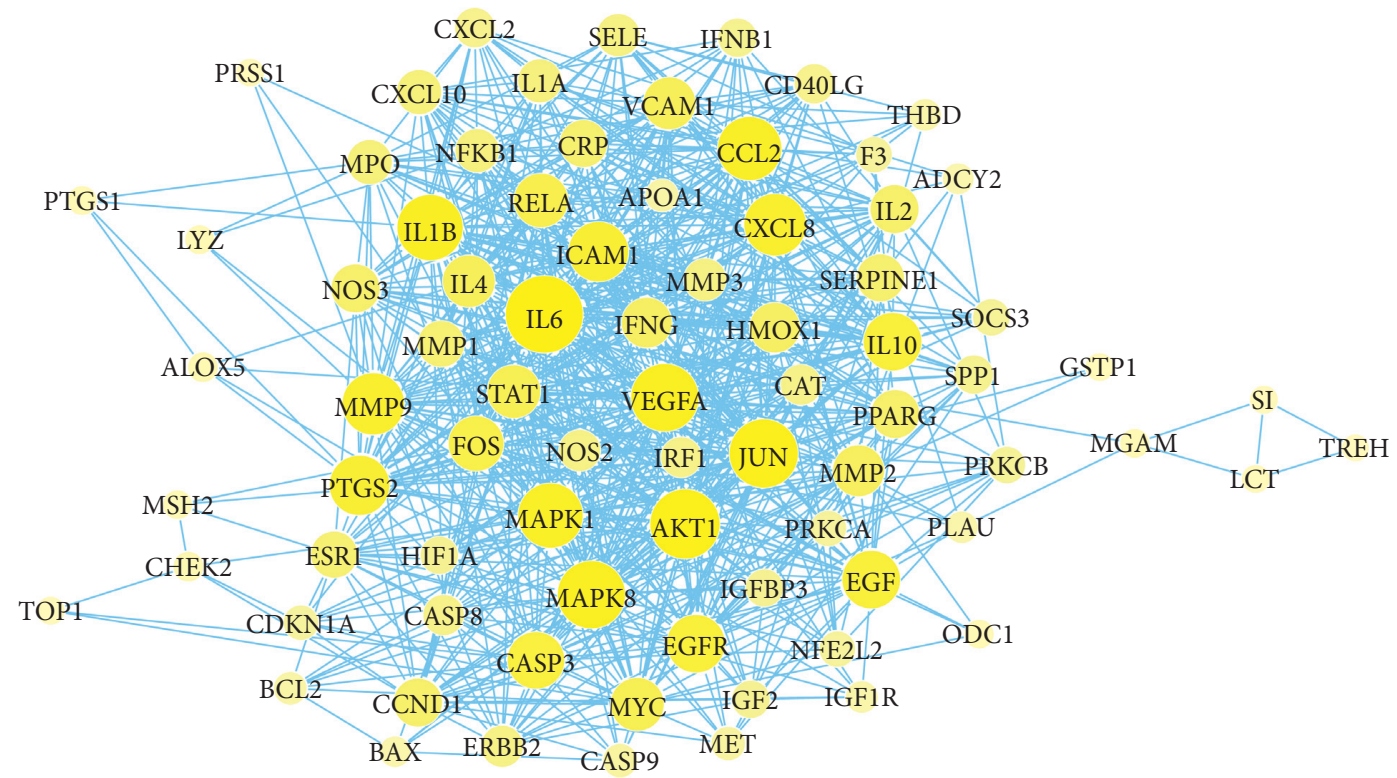

FIGURE 3: The PPI network of the common targets of the selected bioactive compounds and IC-correlated diseases. The larger the yellow nodes and the darker the blue edges, the higher the centrality of the nodes in the network. 


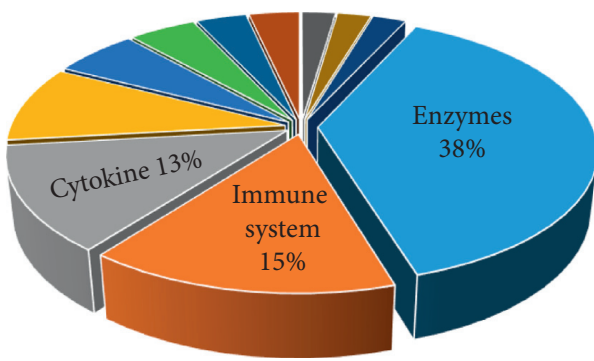

- Enzymes

- Cytokine

- Binding protein

- Unclassificated

- Transporters

- Transporters

\begin{abstract}
- Immune system
- Transcription

- Signaling protein

- Hormone

- Orther
\end{abstract}

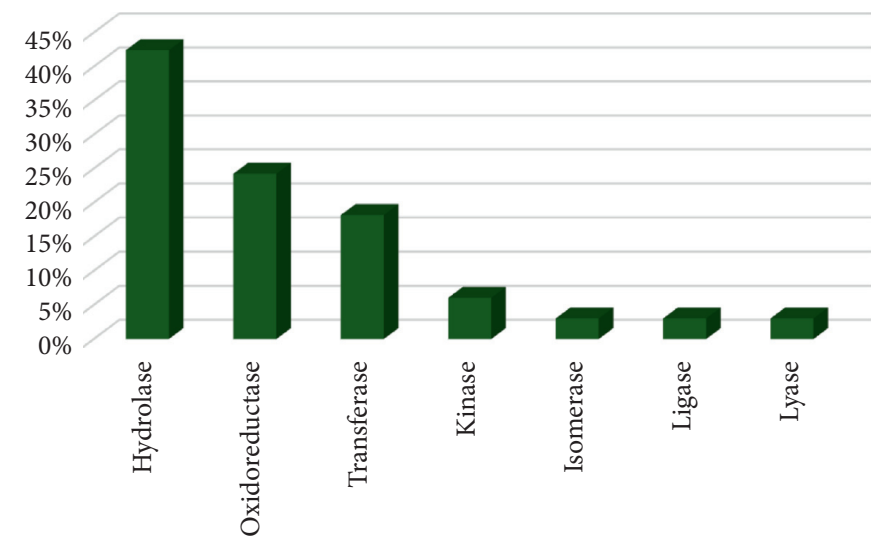

(b)

(a)

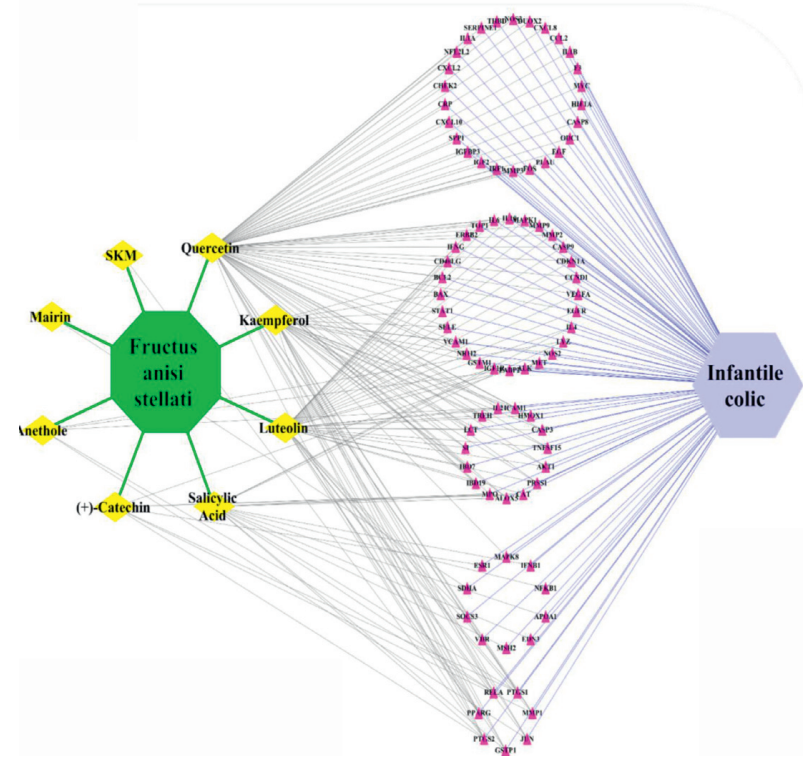

(c)

Figure 4: Compound-target (C-T) network analysis. (a) Distribution of biochemical categories among the common targets. (b) Classification of targets in the enzyme group. (c) C-T network, where FAS is colored green, the compounds are colored yellow, the targets are colored red, and the disease is colored purple.

TABLE 2: The KEGG enrichment analysis for IC-correlated pathways.

\begin{tabular}{lcc}
\hline $\begin{array}{l}\text { Pathway } \\
\text { ID }\end{array}$ & Description & Gene ID \\
\hline hsa04060 & Cytokine-cytokine receptor & IL4, IFNB1, TNFSF15, IL1B, IL10, IL6, IL2, IFNG, CD40LG, CCL2, CXCL8, IL1A, \\
hsa05321 & Inflammatory bowel disease (IBD) & CXCL11, CXCL2, CXCL10 \\
hsa04972 & $\begin{array}{c}\text { Pancreatic secretion } \\
\text { hsa04672 }\end{array}$ & RELA, IL4, NFKB1, IL1B, IL10, JUN, IL6, IL2, IFNG, STAT1, IL1A \\
Intestinal immune network for IgA & CHRM3, CTRB1, PRSS3, PRSS1, ADCY2, PRKCA, PRKCB \\
hsa04973 & Carboduction & IL4, IL10, IL6, IL2, CD40LG \\
hsa00052 & absorption \\
hsa00500 & Galactose metabolism & AKT1, PRKCB, MGAM, HK2 \\
\hline
\end{tabular}


TABLE 3: The IC-correlated diseases from KEGG database.

\begin{tabular}{lc}
\hline Disease ID & Description \\
\hline H00070 & Galactosemia \\
H00115 & Congenital sucrase-isomaltase deficiency; disaccharide intolerance I \\
H00116 & Congenital lactase deficiency; disaccharide intolerance II \\
H00286 & Crohn disease \\
H00408 & Type 1 diabetes mellitus \\
H00409 & Type 2 diabetes mellitus \\
H01227 & Inflammatory bowel disease (IBD) \\
H01261 & Congenital glucose-galactose malabsorption \\
H01466 & Ulcerative colitis \\
H02008 & Galactose-1P uridylyltransferase deficiency; classic galactosemia \\
H02090 & Trehalase deficiency \\
H02330 & Pancreatic lipase deficiency \\
\hline
\end{tabular}

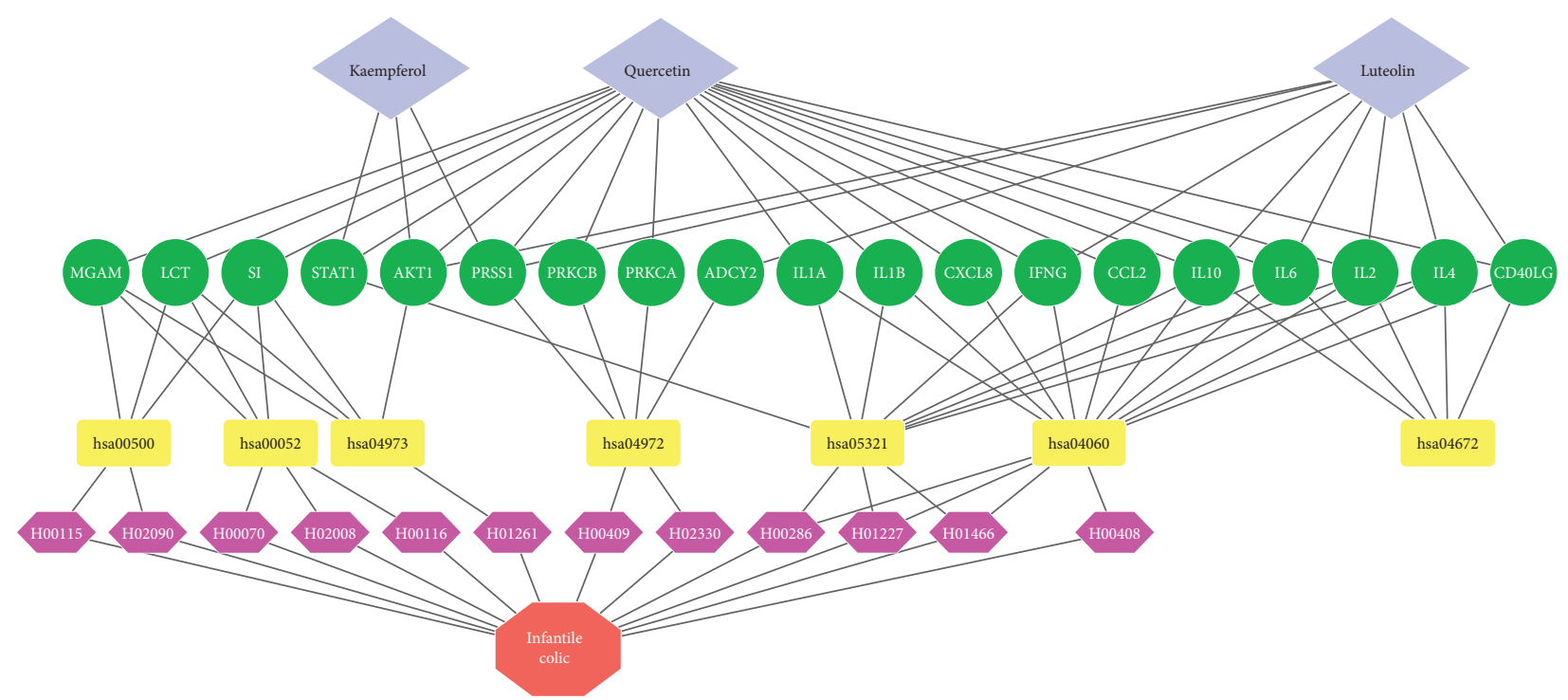

Figure 5: The compound-target-pathway-disease (C-T-P-D) network. In the first row, the gray diamonds represent the compounds, the second-row green ellipses represent the targets, the third-row yellow rectangles represent the biological pathways, the fourth-row pink hexagons represent the diseases, and the fifth-row red octagon represents the infantile colic.

(H00116), and galactose-1P uridylyltransferase deficiency (H02008), whereas hsa00500 is connected to congenital sucrase-isomaltase deficiency (H00115) and trehalase deficiency (H02090); and hsa04972 is related to Type 2 diabetes mellitus (H00409) and pancreatic lipase deficiency (H02330). Module II consisted of two pathways (hsa05321 and hsa04672) related to Crohn disease (H00286), IBD (H01227), and ulcerative colitis (H01466). Module III consisted of one pathway (hsa04060) related to cytokinecytokine receptor interaction and associated with Crohn disease (H00286), Type 1 diabetes mellitus (H00408), IBD (H01227), and ulcerative colitis (H01466).

3.7. C-T-P-D Network Analysis. The C-T-P-D network (Figure 5) was constructed using Cytoscape with 3 key compounds, 12 IC-correlated diseases, 7 relevant molecular pathways, and 19 targets associated with the molecular pathways. As shown in Figure 5, each pathway in the network regulates one or more IC-correlated diseases. Some protein targets (i.e., SI, LCT, MGAM, IL6, IL4, IL2, and IL10) are shared by multiple pathways. Furthermore, the three key compounds, quercetin, luteolin, and kaempferol, bind to 18 (94.7\%), 9 (47.4\%), and $3(15.8 \%)$ out of the 19 targets suggesting the key therapeutic role of quercetin, and the supporting roles of luteolin and kaempferol in treating IC.

3.8. Molecular Docking. The bindings of quercetin, luteolin, and kaempferol to 19 targets were further verified by molecular docking with SystemsDock. The binding strength of a target-compound complex was evaluated with a docking score. As shown in Table 4, all 48 combinations of targetcompound pairs showed docking scores more than a cutoff value at 5.52, indicating strong binding between a compound ligand and a protein target [20]. These results further confirmed that quercetin, luteolin, and kaempferol are the key ligand to the targets with good binding properties.

3.9. Supporting Evidence for FAS Key Compounds as Therapeutic Agents. Quercetin was reported to possess antiulcer, 
TABle 4: The docking scores obtained by SystemsDock for the binding interactions between target proteins and key compounds.

\begin{tabular}{|c|c|c|c|c|}
\hline No. & $\begin{array}{l}\text { Protein } \\
\text { name }\end{array}$ & $\begin{array}{l}\mathrm{PDB} \\
\mathrm{ID}^{\mathrm{a}}\end{array}$ & $\begin{array}{c}\text { Test } \\
\text { compounds }\end{array}$ & $\begin{array}{c}\text { Docking scores } \\
\left(\mathrm{pK}_{\mathrm{d}}\right)^{\mathrm{b}}\end{array}$ \\
\hline 1 & STAT1 & 1BF5 & Luteolin & 7.897 \\
\hline 2 & STAT1 & 1BF5 & Kaempferol & 7.875 \\
\hline 3 & STAT1 & 1BF5 & Quercetin & 7.810 \\
\hline 4 & IL1A & 5UC6 & Luteolin & 7.696 \\
\hline 5 & IL1A & 5UC6 & Quercetin & 7.576 \\
\hline 6 & IL1A & 5UC6 & Kaempferol & 7.523 \\
\hline 7 & IL10 & $2 \mathrm{ILK}$ & Luteolin & 7.311 \\
\hline 8 & ADCY2 & $1 \mathrm{AB} 8$ & Kaempferol & 7.057 \\
\hline 9 & ADCY2 & $1 \mathrm{AB} 8$ & Luteolin & 6.989 \\
\hline 10 & ADCY2 & $1 \mathrm{AB} 8$ & Quercetin & 6.982 \\
\hline 11 & IL10 & 2ILK & Kaempferol & 6.779 \\
\hline 12 & IL10 & 2ILK & Quercetin & 6.721 \\
\hline 13 & CCL2 & $1 \mathrm{DOK}$ & Kaempferol & 6.690 \\
\hline 14 & IL6 & $1 \mathrm{ALU}$ & Quercetin & 6.684 \\
\hline 15 & IFNG & $6 \mathrm{E} 3 \mathrm{~K}$ & Quercetin & 6.680 \\
\hline 16 & IFNG & $6 \mathrm{E} 3 \mathrm{~K}$ & Kaempferol & 6.678 \\
\hline 17 & IL2 & 5UTZ & Kaempferol & 6.677 \\
\hline 18 & SI & $3 \mathrm{LPO}$ & Kaempferol & 6.672 \\
\hline 19 & IFNG & $6 \mathrm{E} 3 \mathrm{~K}$ & Luteolin & 6.670 \\
\hline 20 & IL1B & $5 \mathrm{MVZ}$ & Quercetin & 6.656 \\
\hline 21 & CD40LG & $3 \mathrm{LKJ}$ & Luteolin & 6.655 \\
\hline 22 & IL2 & 5UTZ & Quercetin & 6.655 \\
\hline 23 & IL2 & $5 \mathrm{UTZ}$ & Luteolin & 6.652 \\
\hline 24 & IL1B & $5 \mathrm{MVZ}$ & Kaempferol & 6.647 \\
\hline 25 & IL6 & $1 \mathrm{ALU}$ & Kaempferol & 6.644 \\
\hline 26 & CCL2 & $1 \mathrm{DOK}$ & Quercetin & 6.624 \\
\hline 27 & AKT1 & $3 \mathrm{OCB}$ & Quercetin & 6.622 \\
\hline 28 & IL6 & $1 \mathrm{ALU}$ & Luteolin & 6.622 \\
\hline 29 & CD40LG & $3 \mathrm{LKJ}$ & Quercetin & 6.610 \\
\hline 30 & IL1B & $5 \mathrm{MVZ}$ & Luteolin & 6.608 \\
\hline 31 & PRKCB & $2 \mathrm{I} 0 \mathrm{E}$ & Luteolin & 6.607 \\
\hline 32 & CCL2 & $1 \mathrm{DOK}$ & Luteolin & 6.599 \\
\hline 33 & AKT1 & $3 \mathrm{OCB}$ & Luteolin & 6.530 \\
\hline 34 & CD40LG & $3 \mathrm{LKJ}$ & Kaempferol & 6.511 \\
\hline 35 & PRKCA & 4RA4 & Kaempferol & 6.452 \\
\hline 36 & PRKCA & 4RA4 & Luteolin & 6.442 \\
\hline 37 & AKT1 & $3 \mathrm{OCB}$ & Kaempferol & 6.436 \\
\hline 38 & PRKCA & 4RA4 & Quercetin & 6.416 \\
\hline 39 & SI & $3 \mathrm{LPO}$ & Quercetin & 6.415 \\
\hline 40 & SI & $3 \mathrm{LPO}$ & Luteolin & 6.397 \\
\hline 41 & PRKCB & $2 \mathrm{IOE}$ & Quercetin & 6.359 \\
\hline 42 & IL4 & $5 \mathrm{FHX}$ & Quercetin & 6.301 \\
\hline 43 & IL4 & 5FHX & Luteolin & 6.265 \\
\hline 44 & PRKCB & $2 \mathrm{IOE}$ & Kaempferol & 6.256 \\
\hline 45 & IL4 & 5FHX & Kaempferol & 6.156 \\
\hline 46 & PRSS1 & 4WWY & Luteolin & 6.044 \\
\hline 47 & PRSS1 & 4WWY & Kaempferol & 5.939 \\
\hline 48 & PRSS1 & 4WWY & Quercetin & 5.850 \\
\hline
\end{tabular}

${ }^{a}$ PDB ID, protein data bank identifier. ${ }^{b} \mathrm{pK}_{\mathrm{d}}$, the negative logarithm of dissociation constant.

antioxidant, antidiabetic, and anti-inflammatory properties $[24,25]$ and could modulate some key regulatory enzymes in humans such as alkaline phosphatase and lens aldose reductase [26]. Besides, it was reported that quercetin can increase the expression of lactase [27] and inhibit the release of proinflammatory mediators and the expression of inflammatory proteins like adhesion molecules, cyclooxygenase, and nitric oxide synthase [28]. Recent research shows that quercetin can increase the secretion of immunoglobulin $A$ (IgA) [29]. Quercetin could affect the progression of colitis and IBD [30] and possessed protective and beneficial effects on chronic intestinal inflammation [31]. It was used to treat inflammatory illnesses caused by mast cells $[32,33]$, treat IBD induced by Citrobacter rodentium [34], and effectively decrease oxidative stress and inflammatory damage to both ileum and colon tissues [35]. Luteolin was reported to have strong anti-inflammatory activity [36, 37] and strong radical scavenging and cell-protective properties [38]. It was considered as a therapeutic agent for IBD [39], and inflammation-related diseases in humans [40]. Kaempferol also showed anti-inflammatory activities and immunomodulatory effects [41].

\section{Conclusion}

In this work, we have investigated the molecular mechanisms of FAS for the treatment of IC with a network pharmacology approach. The active compounds of FAS were selected through the ADME screening of the FAS compounds from the TCMSP database. The common targets of the active compounds and IC-correlated diseases were obtained from PharmMapper and GeneCards and used to construct the PPI network. Through GO and KEGG enrichment analyses, the seven molecular pathways that were associated with three key compounds, 19 targets, and 12 ICcorrelated diseases were extracted and used to construct the C-T-P-D network. The network analysis revealed that FAS compound quercetin is the key therapeutic agent, whereas luteolin and kaempferol are the regulating and modulating agents in treating IC. The therapeutic effect of FAS on IC was based on the synergistic effect of multiple compounds acting on multiple targets through various therapeutic modules including the inhibition of inflammatory reactions, stimulating IgA production in the gastrointestinal tract, and enhancing the secretion of digestive enzymes. Our findings were supported by the molecular docking analysis, and the experimental results from the literature search. This work provides a mechanistic guide for preclinical and clinical studies of FAS on IC therapy.

\section{Data Availability}

The data used to support the findings of this study are available from the corresponding author upon request.

\section{Conflicts of Interest}

The authors declare that they have no conflicts of interest.

\section{Authors' Contributions}

Xingyu Li performed data acquisition and network pharmacology analysis and wrote the manuscript and submitted it. Yan Xu designed and supervised the study and conducted the manuscript revision. 


\section{Acknowledgments}

This work was supported by the National Natural Science Foundation of China (Grant no. 31660536) and the China Scholarship Council (Grant no. 201808535031) to Xingyu Li.

\section{Supplementary Materials}

Table S1: the ADME parameters of the identified components of FAS and their chemical structures; Table S2: the predicted binding proteins and gene symbols of eight selected bioactive components of FAS; Table S3a: the genes of IC-associated diseases obtained from GenCards database; Table S3b: the genes of IC-associated diseases obtained from OMIM database; Table S4: key information of the common genes obtained by intersecting the genes of component binding proteins and IC-associated diseases; Table S5: the topological features of all nodes in the IC targets' PPI network constructed by string website; Table S6: the GO molecular function enrichment analysis for selected targets; Table S7. the KEGG pathway enrichment analysis for selected targets; Table S8: the KEGG pathways and all related diseases. (Supplementary Materials)

\section{References}

[1] J. Zeevenhooven, P. D. Browne, M. P. L'Hoir, C. de Weerth, and M. A. Benninga, "Infant colic: mechanisms and management," Nature Reviews Gastroenterology \& Hepatology, vol. 15, no. 8, pp. 479-496, 2018.

[2] J. Zeevenhooven, I. J. N. Koppen, and M. A. Benninga, "The new Rome IV criteria for functional gastrointestinal disorders in infants and toddlers," Pediatric Gastroenterology, Hepatology \& Nutrition, vol. 20, no. 1, pp. 1-13, 2017.

[3] F. Ghorat, B. Afzali, R. Akrami, F. Ghorat, Z. Niktabe, and M. Sahebkar, "Effect of topical chamomile oil (Matricaria chamomile $L$ ) as a supplementary method for improving colic symptoms in infants: a randomized placebo-controlled clinical trial," Iranian Journal of Neonatology, vol. 10, no. 1, pp. 15-22, 2019.

[4] H. Makrane, M. Aziz, M. Berrabah et al., "Myorelaxant activity of essential oil from Origanum majorana L. on rat and rabbit," Journal of Ethnopharmacology, vol. 228, pp. 40-49, 2019.

[5] M. Gh, M. Ghazanfarpour, L. Kargarfard, and M. Babakhanian, "Effectiveness of herbal medicines containing phytoestrogens to treat infantile colic: a meta-analysis review," Journal of Pediatrics, vol. 7, no. 1, pp. 1-10, 2019.

[6] M. W. Chase, M. Christenhusz, M. Fay et al., "An update of the Angiosperm Phylogeny Group classification for the orders and families of flowering plants: apg IV," Botanical Journal of the Linnean Society, vol. 181, no. 1, pp. 1-20, 2016.

[7] W. Peng, Z. Lin, L. Wang, J. Chang, F. Gu, and X. Zhu, "Molecular characteristics of Illicium verum extractives to activate acquired immune response," Saudi Journal of Biological Sciences, vol. 23, no. 3, pp. 348-352, 2016.

[8] X. Zhang, X. Meng, J. Wu, L. Huang, and S. Chen, "Global ecological regionalization of 15 Illicium species: nature sources of shikimic acid," Chinese Medicine, vol. 13, no. 1, p. 31, 2018.
[9] N. Techen, Z. Pan, B. Scheffler, and I. Khan, "Detection offllicium anisatumas Adulterant offllicium verum," Planta Medica, vol. 75, no. 04, pp. 392-395, 2009.

[10] R. Paul and R. Geetha, "Evaluation of anti-inflammatory action of Illicium verum-An in vitro study," Drug Invention Today, vol. 10, no. 12, pp. 2441-2444, 2018.

[11] J. Kamoun, R. Rahier, M. Sellami et al., "Identification of a new natural gastric lipase inhibitor from star anise," Food \& Function, vol. 10, no. 1, pp. 469-478, 2019.

[12] C. Zhou, L. Liu, J. Zhuang et al., "A systems biology-based approach to uncovering molecular mechanisms underlying effects of traditional Chinese medicine qingdai in chronic myelogenous leukemia, involving Integration of network pharmacology and molecular docking technology," Medical Science Monitor, vol. 24, pp. 4305-4316, 2018.

[13] J. Ru, P. Li, J. Wang et al., "TCMSP: a database of systems pharmacology for drug discovery from herbal medicines," Journal of Cheminformatics, vol. 6, no. 1, p. 13, 2014.

[14] X. Zhang, W. Xiao, X. Xu et al., "Molecular mechanism of Guizhi Fuling Capsule for treatment of dysmenorrhea, pelvic inflammatory disease, and hysteromyoma via network pharmacology," Chinese Traditional and Herbal Drugs, vol. 47, no. 1, pp. 81-94, Article ID 0253-2670, 2016.

[15] X. Wang, Y. Shen, S. Wang et al., "PharmMapper 2017 update: a web server for potential drug target identification with a comprehensive target pharmacophore database," Nucleic Acids Research, vol. 45, no. 1, pp. W356-W360, 2017.

[16] G. Stelzer, N. Rosen, I. Plaschkes et al., "The GeneCards suite: from gene data mining to disease genome sequence analyses," Current Protocols in Bioinformatics, vol. 54, no. 1, 2016.

[17] J. S. Amberger, C. A. Bocchini, F. Schiettecatte, A. F. Scott, and A. Hamosh, "OMIM.org: online Mendelian Inheritance in Man (OMIM), an online catalog of human genes and genetic disorders," Nucleic Acids Research, vol. 43, no. D1, pp. D789-D798, 2014.

[18] S. K. Burley, H. M. Berman, C. Bhikadiya et al., "RCSB Protein Data Bank: biological macromolecular structures enabling research and education in fundamental biology, biomedicine, biotechnology and energy," Nucleic Acids Research, vol. 47, no. D1, pp. D464-D474, 2018.

[19] R. C. Gentleman, V. J. Carey, D. M. Bates et al., "Bioconductor: open software development for computational biology and bioinformatics," Genome Biology, vol. 5, no. 10, p. R80, 2004.

[20] K.-Y. Hsin, Y. Matsuoka, Y. Asai et al., "systemsDock: a web server for network pharmacology-based prediction and analysis," Nucleic Acids Research, vol. 44, no. 1, pp. W507-W513, 2016.

[21] V. Marinov and S. Valcheva-Kuzmanova, "Review on the pharmacological activities of anethole," Scripta Scientifica Pharmaceutica, vol. 2, no. 2, pp. 14-19, 2015.

[22] M. Ahmed, A. G. Billoo, K. Iqbal, and A. Memon, "Clinical efficacy of lactase enzyme supplement in infant colic: a randomised controlled trial," The Journal of the Pakistan Medical Association, vol. 68, no. 12, pp. 1744-1747, 2018.

[23] T. Vezza, A. Rodríguez-Nogales, F. Algieri, M. Utrilla, M. Rodriguez-Cabezas, and J. Galvez, "Flavonoids in inflammatory bowel disease: a review," Nutrients, vol. 8, no. 4, p. 211, 2016.

[24] D. Dodda, R. Chhajed, and J. Mishra, "Protective effect of quercetin against acetic acid induced inflammatory bowel disease (IBD) like symptoms in rats: possible morphological and biochemical alterations," Pharmacological Reports, vol. 66, no. 1, pp. 169-173, 2014.

[25] A. During and Y. Larondelle, "The O-methylation of chrysin markedly improves its intestinal anti-inflammatory 
properties: structure-activity relationships of flavones," Biochemical Pharmacology, vol. 86, no. 12, pp. 1739-1746, 2013.

[26] R. Ramachandra, A. K. Shetty, and P. V. Salimath, "Quercetin alleviates activities of intestinal and renal disaccharidases in streptozotocin-induced diabetic rats," Molecular Nutrition \& Food Research, vol. 49, no. 4, pp. 355-360, 2005.

[27] C. Magkoufopoulou, S. M. H. Claessen, D. G. J. Jennen, J. C. S. Kleinjans, and J. H. M. Van Delft, "Comparison of phenotypic and transcriptomic effects of false-positive genotoxins, true genotoxins and non-genotoxins using HepG2 cells," Mutagenesis, vol. 26, no. 5, pp. 593-604, 2011.

[28] S. Habtemariam and A. Belai, "Natural therapies of the inflammatory bowel disease: the case of rutin and its aglycone, quercetin," Mini Reviews in Medicinal Chemistry, vol. 18, no. 3, pp. 234-243, 2018.

[29] J. X. Yang, T. C. Maria, B. Zhou et al., "Quercetin improves immune function in Arbor Acre broilers through activation of NF- $\kappa$ B signaling pathway," Poultry Science, vol. 99, no. 2, pp. 906-913, 2020.

[30] C. F. S. Guazelli, V. Fattori, B. B. Colombo et al., "Quercetinloaded microcapsules ameliorate experimental colitis in mice by anti-inflammatory and antioxidant mechanisms," Journal of Natural Products, vol. 76, no. 2, pp. 200-208, 2013.

[31] I. Castangia, A. Nácher, C. Caddeo et al., "Therapeutic efficacy of quercetin enzyme-responsive nanovesicles for the treatment of experimental colitis in rats," Acta Biomaterialia, vol. 13, pp. 216-227, 2015.

[32] Y.-D. Min, C.-H. Choi, H. Bark et al., "Quercetin inhibits expression of inflammatory cytokines through attenuation of NF- $\kappa \mathrm{B}$ and p38 MAPK in HMC-1 human mast cell line," Inflammation Research, vol. 56, no. 5, pp. 210-215, 2007.

[33] B. Romier, Y.-J. Schneider, Y. Larondelle, and A. During, "Dietary polyphenols can modulate the intestinal inflammatory response," Nutrition Reviews, vol. 67, no. 7, pp. 363-378, 2009.

[34] R. Lin, M. Piao, and Y. Song, "Dietary quercetin increases colonic microbial diversity and attenuates colitis severity in Citrobacter rodentium-Infected mice," Frontiers in Microbiology, vol. 10, p. 1092, 2019.

[35] Ö. Pişkin, B. G. Aydın, Y. Baş et al., "Protective effects of quercetin on intestinal damage caused by ionizing radiation," Haseki Tpp Bülteni, vol. 56, no. 1, pp. 14-21, 2018.

[36] N. Aziz, M.-Y. Kim, and J. Y. Cho, "Anti-inflammatory effects of luteolin: a review of in vitro, in vivo, and in silico studies," Journal of Ethnopharmacology, vol. 225, pp. 342-358, 2018.

[37] S. Wang, M. Cao, S. Xu et al., "Luteolin alters macrophage polarization to inhibit inflammation," Inflammation, vol. 43, no. 1, pp. 95-108, 2020.

[38] Y. Li, L. Shen, and H. Luo, "Luteolin ameliorates dextran sulfate sodium-induced colitis in mice possibly through activation of the Nrf2 signaling pathway," International Immunopharmacology, vol. 40, pp. 24-31, 2016.

[39] C. Nunes, L. Almeida, R. M. Barbosa, and J. Laranjinha, "Luteolin suppresses the JAK/STAT pathway in a cellular model of intestinal inflammation," Food \& Function, vol. 8, no. 1, pp. 387-396, 2017.

[40] T. Boeing, P. De Souza, S. Speca et al., "Luteolin prevents irinotecan-induced intestinal mucositis in mice through antioxidant and anti-inflammatory properties," British Journal of Pharmacology, vol. 177, pp. 1-16, 2020.

[41] M.-Y. Park, G. E. Ji, and M.-K. Sung, "Dietary kaempferol suppresses inflammation of dextran sulfate sodium-induced colitis in mice," Digestive Diseases and Sciences, vol. 57, no. 2, pp. 355-363, 2012. 